\title{
Logistics Management Analysis for the Wafer Foundry Supply Chain
}

\author{
Yu-Chuan Liu \\ Department of Information Management \\ Tainan University of Technology \\ Tainan, Taiwan \\ e-mail: t00258@mail.tut.edu.tw
}

\author{
Hong-Mei Gao \\ Department of Economic Management \\ Tianjin Agricultural University \\ Tianjin, China \\ e-mail: gaohongmei@126.com
}

\begin{abstract}
The IC foundry business had been remarkably developed for the past decade in Taiwan. The well-known IC design house, the wafer fabrication industries, and the IC packaging/testing business had together formed a contiguous supply chain form material to system. Logistic management of wafers is the key linkage in the IC foundry supply chain. The objectives of this paper include the issues review and solution requirements analysis for the global wafer logistics. The model-driven business transformation, an approach uses formal models to explicitly define the structure and behavior of a business, is applied for the review and analysis process. Specific requirements for the wafer logistic management solutions are proposed. Development of this research is crucial for the solution integration of information and logistic management in IC foundry business.
\end{abstract}

Keywords-logistics management; information management system; supply chain management; model-driven-business transformation; wafer foundry.

\section{INTRODUCTION}

Supply chains are complicated dynamical systems triggered by customer demands. Proper selection of equipment, machinery, buildings and transportation fleets is a key component for the success of such systems. However, efficiency of supply chains mostly depends on management decisions, which are often based on intuition and experience [1]. In the current marketplace companies are competing in a way of supply chain coordination. To keep up in this high competitive market companies tend to concentrate on their core activities and outsource more and more activities. Supply chain coordination should not only relate to the data exchange between enterprises, but also to the further extension of business process sharing. The main interest is to establish the right organizational interfaces within the supply chain companies, often located in different countries with different backgrounds and standards, to produce these new and competitive products. Keeping track of product data of these 'innovative' products over their individual lifecycle becomes a challenging endeavor [2].

Semiconductor manufacturing business in Taiwan is worldwide famous by developing a contiguous IC foundry supply chain from material to system. The manufacturing supply chain provides foundry services for those IC design houses do not possess any manufacturing capacity. Leading enterprises in this supply chain, including TSMC,
UMC, ASE, ... etc., had already proposed an unified B2B e-business process, by RosettaNet PIPs standards, for the supply chain management in IC foundry [3-5]. This procedure had become a world-wide standard for the semiconductor foundry business. Contrary to the independent IC design houses, most IC manufacturing were originally completed by the integrated device manufacturers (IDM), such as TI, INTEL, SAMAUNG, ...etc. The IDMs design their own IC products and finish the manufacturing process with their own production factories. As the foundry business provide integrated IC design, wafer fabrication, and IC packaging services, the IDM companies are continuously releasing the manufacturing order to foundry. By integrating the shop flow control system, ERP, and SCM systems, the supply chain venders can provide customers a unified and instant service for order checking, order scheduling ..., etc. Taiwan's semiconductor manufacturing enterprises are expected to be further linked with the international semiconductor business, no matter IDM or design houses, and hence keep playing the leading roles in the wafer foundry related business.

As the manufacturing process is no longer completed in a single IDM company, half-finished wafers have to be transported worldwide. The business process sharing and/or integration become inevitable for IC foundry business. Logistics and information management of the wafer are the key linkages in the IC foundry process. The complexity of wafer information management is due to the complicated customer and product requirements. Foundry customers will order their wafer manufacturing process in two manners, one is to authorize the wafer fabrication vender to handle the whole process until final IC products shipped, and the other is to control every manufacturing supply chain venders by themselves. Different types of order authorization will be applied for all kinds of products and cause different characteristics and requirements. The customers of the IC packaging FAB include not only the wafer fabrication FAB but also the IC design houses and the end users. Unfortunately, there is no rule between the products, customers, and foundry orders. The same foundry product can belongs to different customers for the packaging foundry. The complicated customer and product relationships cause not only the incorrect customer judgment; the error information also plagues the product traceability and production scheduling in the supply chain. 
To provide solutions for a problem domain, system analysis by constructing models is widely applied. The model-driven approaches to the realization of solutions for today's enterprise systems were presented in [6]. An everincreasing trend in today's firms is to exploit outsourcing for those information systems (IS) functions deemed to be outside the company's core competence. Given the multiattribute nature of IS outsourcing decision, six factors, including economics, resource, strategy, risk, management and quality, were suggested to be considered for outsourcing decisions [7]. Gong and Shei [8] employed the IDEF0 analysis together with the object-oriented and the distributed control architecture for the modeling of shop flow control information system in an IC packaging FAB. Current researches mostly emphasized on the analysis, rationalization, modeling, and implementation for the production process of IC packaging FAB. The wafer logistics and information management, i.e., the pre-process of IC packaging process, is mainly relied on manual management. Hill et al [9] mentioned that the greatest productivity gains in business processes have been achieved by formalizing the processes into computermanaged workflows. A full life-cycle business-totechnology method, model-driven business transformation MDBT [10], is both a business transformation methodology and a set of innovative technologies that allow business strategies to be realized by choreographing workflow tools and human activities. MDBT uses formal models to explicitly define the structure and behavior of a business component. Kumaran et al [11] presented a new approach to IT service workflow automation and a new generation of service-delivery management systems based on the model-driven transformational approach and service-oriented architecture.

The wafer logistics and information flow play an important role in the IC foundry supply chain in Taiwan. The efficiency of the supply chain system will be degraded if the process and/or information integration between the supply chain venders can not be effectively solved. System requirements for the information management of IC packaging business had been studied in [12]. Based on the previous analysis results of information management for IC packaging enterprises, the objective of this paper is to further provide a complete study on the issues and solutions for wafer logistics management in the IC foundry supply chain. The MDBT approach is applied to propose a complete and integrated solution. Development of this research is crucial for the solution integration of information and logistic management in IC foundry business.

\section{ISSUE ANALYSIS FOR WAFER FOUNDRY LOGISTICS}

The structured problem solving cycle started from conceptualizing the problem situation and specifying the relevant parts of the problem situation. The current situation of the IC foundry business is firstly reviewed. Issues of wafer transportation, customer and product information, and wafer handling management are discussed in the following sub-sections. MDBT approach is then briefly introduced and applied for the solution analysis in section III. A brief conclusion is given in section IV.

\section{A. Wafer Transportation}

Venders in the IC foundry supply chain are located in different areas of Taiwan. The wafer FABs are located mainly in the north and the IC packaging FAB are distributed in the central and south of Taiwan. Wafer receiving for IC package $\mathrm{FAB}$ can be summarized in three manners. The first type is received directly from the wafer FAB. The warehouse department of IC packaging FAB goes directly to the wafer FAB to receive the wafer. Take Taiwan's supply chain as an example, the packaging manufacturer ASE in Kaoshong send their trucks to TSMC in Shinchu or Tainan to receive the wafers to be packaged. The second type of wafer receiving is received from the custom. The warehouse department of IC packaging FAB goes to the customs to receive the wafer from overseas customers. The third type is received from express delivery. In some urgent cases, the customers send their wafers to the warehouse of IC packaging FAB directly by express delivery such as DHL. The quality of transportation and wafer status can not be guaranteed especially for the second the third types of transportation. When the IC packaging FAB receives damaged wafers, it is difficult to judge the responsibility belongings. The first type of transportation is mainly controlled by the IC packaging $\mathrm{FAB}$, however, the similar situation of un-clarified damage can still happen. As Taiwan's $300 \mathrm{~mm}$ wafer Fabrication and packaging technologies successfully developed and improved, more and more $300 \mathrm{~mm}$ wafers will be transported either by highway or by air cargo for foundry purpose. Because the wafer area is significantly enlarged and the thickness is reduced due to grounding process, more damages can be induced during transportation.

SEMI, Semiconductor Equipment and Material International, had long been focused on the standardization of wafer manufacturing process. The wafer box related standards, such as E1.9-0701, E7.1-1101, E75-0698, E1060301 for the $300 \mathrm{~mm}$ front open unified pod, or M29-1296 and M31-0999 for the 300mm front open shipping box, are all focused on the automation interface design in the wafer fabrication FAB. The vibration and shock protection during transportation are not considered. The only wafer protection during transportation is the packing carton. Due to the globalization of semiconductor manufacturing, wafers have to be transported overseas from wafer fabrication FAB to the IC packaging FAB. The protection of wafers during transportation is imperative to be solved.

\section{B. Customer and Product Information}

In general, IC design house or end user will order their wafer manufacturing process in two manners, one is to authorize the wafer Fabrication vender to handle the whole process and deliver the final IC product, and the other is to predominate every manufacturing supply chain venders. The customers of the IC packaging FAB include not only the wafer fabrication FAB all around the world, but also the IC design house and the end users. There is no rule between the products, customers, and foundry orders. In some cases, the IC design house will control the whole OEM process, i.e., all the manufacturing supply chain venders will face the same end customer. Sometimes, the design house may issue a complete authorized order to wafer Fabrication, the wafer FAB will become the customer for the other manufacturers of the supply chain. 
The semi-finished wafers sent from the same wafer fabrication FAB can hence belong to different customer for the packaging manufacturer. The difference of products and the special environmental characteristic cause the intricate relation among the packaging manufacturer, wafer FAB, and the end customer. The complicated customer relationships cause not only the incorrect customer information for the supply chain management, the error information can also plague the product traceability and production scheduling.

When the wafers arrive at the warehouse of IC packaging $\mathrm{FAB}$, the first step of the receiving process is the customer and product information judgment. The operator of the wafer warehouse should check the corresponding production procedures such as whether need wafer inspection, or transferred directly to the quality control department for warehouse entrance examination, or special requirements that should be handed over to the production line. In addition to the intricate customer information, the production procedures in the IC packaging $\mathrm{FAB}$ also vary in a wide range according to the distinct customer's requirements. The same product from the wafer FAB, i.e., the customer or the semi-finished product provider for the packaging manufacturer, often undergoes different production procedures. For instance, the wafers may need to be pre-inspected before entering the warehouse in some case while the same pre-inspection process can be omitted for the different production lot of the same product. The related product information is mostly transmitted by artificial notification. Lack of instant information updating and rigmarole contrived operations can lead to delay and/or error production routing judgment which will severely degrade the efficiency of the supply chain system. The customer and product information for the production procedure judgment is complicated and sometimes changed un-expectable for the operators. One can not expect artificial operations to meet exact accuracy. Consequently, a real-time and exact information transmission between the supply chain and the production line is imperative to reduce the production mistake and utilize the supply chain efficiency.

\section{Wafer Handling Management}

The wafer management process for IC packaging FAB includes: wafer receiving, warehouse entrance, storage, and production releasing. The wafer receiving for different customers can be summarized as mentioned in section 2.1. When the wafers arrive at the warehouse of IC packaging FAB, operators of the wafer warehouse should check the corresponding production procedures as described in section 2.2. In addition to the wafer receiving process, the warehouse entrance, and production releasing procedures are also visited and examined. There is no lot receiving plan when receiving wafers from customer's site. Operators can neither check the correctness of wafer lot nor the quantity at receiving operation. A particular situation is that the shipping boxes for wafers are not unified yet. Only the shipping information contents are defined by SEMI. The packing manners are not unified. Different wafer FABs will pack their wafers in different shipping boxes even for the same wafers. Some customers use recycled shipping box with labels of the previous lot. Those issues of shipping boxes types and labels received by the same IC packaging FAB will become an obstacle for the efficient and automatic storage management. In addition, wafers are usually asked to be stored under nitrogen environment to prevent the oxidization from mist and oxygen. The IC packaging FAB uses nitrogen cabinets for the storage and management of wafers. A correct inventory check is very difficult for the wafer warehouse of the IC packaging FAB because of the enormous and Rigmarole artificial dockets for those cabinets. The enormous artificial dockets to record the customers, products, and storage data of wafers degrade significantly the efficiency and correctness of wafer handling management. An integrated management for both the shipping boxes and the related storage and product information is yet to be developed.

\section{SOLUTION REQUIREMENTS ANALYSIS BY MDBT}

\section{A. Model-Driven Business Transformation Approach}

Model-driven business transformation, MDBT developed by IBM research, uses formal models to explicitly define the structure and behavior of a business component. These models can be employed to monitor, analyze, and improve its performance, and leverages these models in the construction of its IT systems. The framework is made up of four layers: business strategy, business operations, solution composition, and IT implementation. Each layer constitutes a different level of abstraction, performs a well-defined function, and has a different audience. The strategy layer defines the goals and objectives of the business system. The operation layer describes the operations performed by the business system to achieve the goals. The composition layer is an abstraction of the computational elements that are needed to execute the business operations. The implementation layer specifies how the computational elements are implemented on a specific IT platform. Figure 1 shows the MDBT framework, including the separation of concerns, connections between model layers, and the closed-loop architecture achieved using the BPM component.

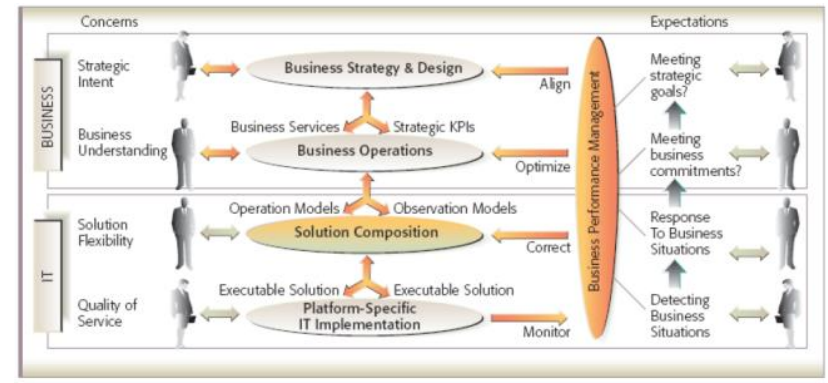

Figure 1. Model-driven business transformation framework, from [11]

In the MDBT approach, the transformation process begins with the identification of the strategic goals and objectives of the business component. This leads to a set of initiatives that support these goals. These initiatives determine the definition, analysis, optimization, and implementation of the business operations of the organization such that the strategic goals can be achieved. Formal definition of the business operations and the operational KPIs (key performance indicators) is the next step of transformation process that was referred as the 
business operation model. A business operation model is different from the more familiar workflow models. A business operation model, on the other hand, defines the key business artifacts and the operations performed on these artifacts. The third step of solution composition in MDBT is the judicious use of technology to support the execution of business operations. This involves the generation of a platform-independent solution composition model and the realization of this model on a specific software platform. The final step in MDBT is to create an implementation of the IT solution on a specific IT platform. Once the solution is deployed, business owners can monitor and analyze business performance using KPIs and continuously improve the models, both at the business and IT levels, based on this performance analysis.

By applying MDBT approach for the solution analysis of wafer logistics management related issues discussed in section II, the definition of goals and objectives for wafer logistics management should firstly analyzed in strategy layer. The issues for wafer logistics management can be summarized in hardware protection and information management. The objectives are consequently to improve the effectiveness and efficiency for wafer logistics management. The KPIs for hardware protection can be wafer loss during transportation and wafer loss during handling. The KPIs for information management can be tact time for wafer receive, tact time for warehouse entrance, through put for warehouse operations, error percentage for customer and product judgment, and cycle time for production release. The business operation and solution composition analysis will be shown in the following section.

\section{B. Business Operation Analysis for Wafer Logistics}

The second step of business operation analysis for MDBT is studied in this section. Considering the supply chain complete process of IC foundry, wafer logistics can be classified into transportation phase and handling phase. The transportation phase mainly consists of the longdistance truck traveling and air cargo. The handling phase mainly deals with the short distance movement, such as handling within warehouse and inter-building transportation. The hardware protection and information flow management should be considered in both phases. The planned operations, scenarios, and functional requirements for transportation and handling phase are shown in Figs. 2 and 3. Transportations either by the highway in domestic or by air cargo for overseas supply chain must all be accomplished safely and efficiently. The vibration and shock protection is the main issue in transportation phase. The handling phase of wafers includes the short distance movement within IC packaging FAB and/or the warehouse. The related operations consist of the lot receiving, incoming quality control, storage, and production releasing. The protection solution during wafer handling and storage should be integrated with the automation related hardware system. The information management through the wafer transportation, receiving, handling, and storage needs to be further integrated.

Detail solution requirements for operation analysis shown in Fig. 2 and 3 are further studied. Solution requirements for the global wafer logistics consist of two main areas. The first one is the wafer protection between transportation, handling, and storage. To identify the damage resource and develop an effective protection device for the shock and vibration are the primal issues. The other is the information integration and management between the supply chain venders. The wafer protection and information management issues are further illustrated in the next section.

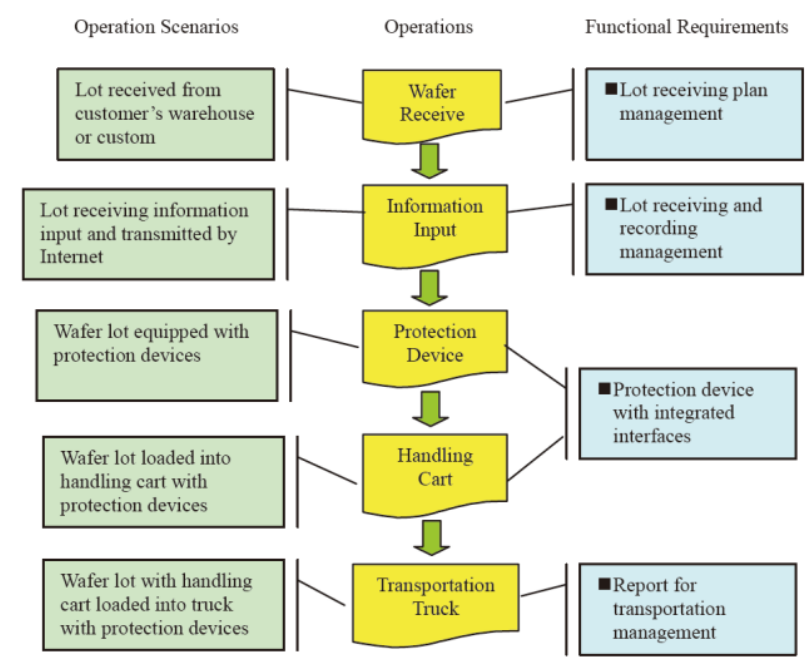

Figure 2. Operation analysis for wafer transportation phase.

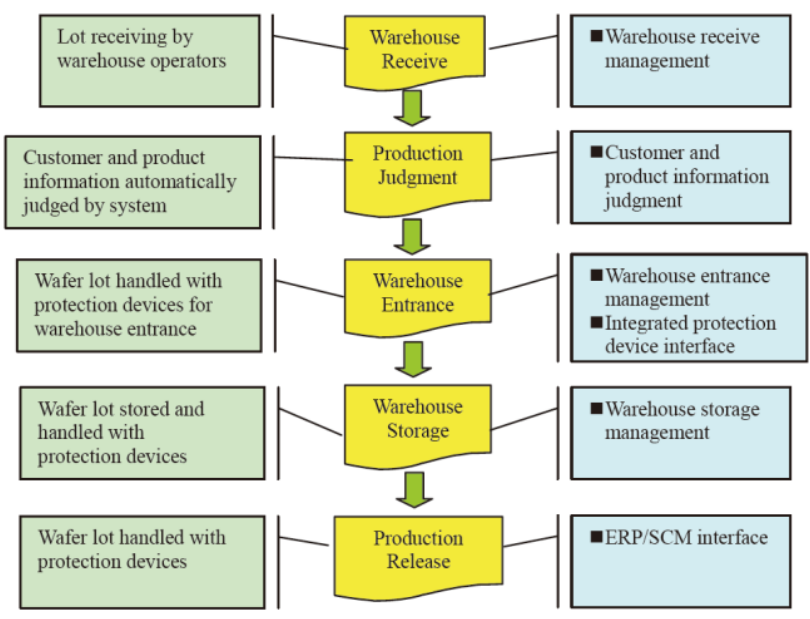

Figure 3. Operation analysis for wafer handling phase.

\section{Solution Composition Analysis for Wafer Logistics}

The wafer logistics process and system analysis for the model of the wafer logistics information management is proposed. Wafer transportation, storage, and handling related management issues are all considered. The current process flow for domestic and overseas wafer transportation is analyzed. The wafer lot receiving, storage, and production releasing procedures are examined. As shown in Fig. 4, the wafer logistics information management system is an integrated interface for the wafer fabrication and IC packaging. The logistical procedures and the information flow between the supply chain enterprises can be shared and integrated for further enhancement of the supply chain management efficiency. The information system is design to cope with the intricate customer relationship, the complicated product 
information, the rigmarole wafer storage and releasing procedures.

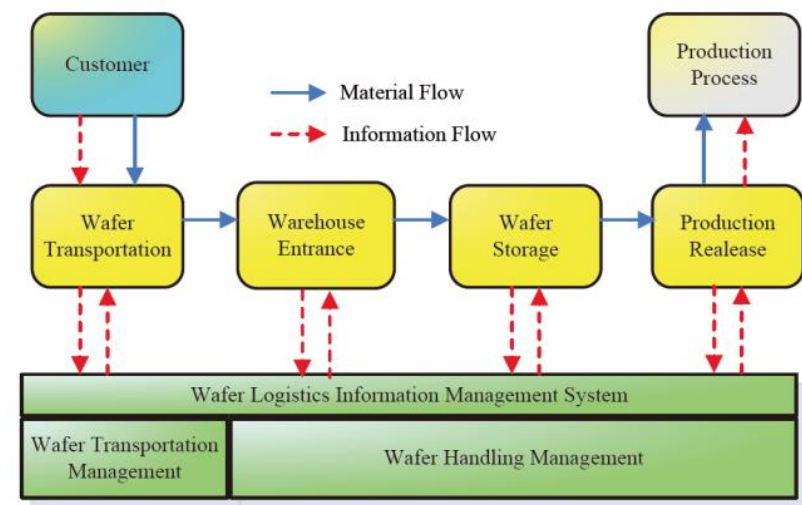

Figure 4. Schematics of the wafer logistics management information system.

The platform independent solution requirements are proposed in this paper. The information system should consist of three major functions, including the information management for wafer transportation, receiving, and storage. The specific functional requirements from configuration management analysis include,

- Wafer transportation management:

In order to instantly record the lot receiving information at customer site, a handhold device to input the information and transmit wirelessly to the server of the system is required. The transportation management functions include: lot receiving and recording, warehouse entrance check and record, and report for transportation management. The related information input needs also the wireless solutions.

- Wafer receiving management:

An interface to input the customer's shipping list is required. A judgment rule data base must be established for to replace the artificial judgment. The judgment rules can be on-line updated. The on-line update authority must belong to the administration level. The wafer receiving management functions include: warehouse entrance record, customer and production procedure judgment, and ERP interface.

- Wafer storage management:

The wafer storage management functions include: warehouse receiving, automatic storage location assignment, load to storage location, unload from storage location, lot split, storage location change, inventory check, and production releasing.

\section{CONCLUSION}

The IC foundry businesses, including the well-known IC design house, the wafer fabrication industries, and IC packaging and testing venders had been remarkably developed in Taiwan. A contiguous IC foundry supply chain from material to system had been successfully integrated. Logistics and information management of the wafer is the key linkage in IC foundry. Because of the importance and complex business characteristics, the issues for wafer logistics and the related information management is imperative. This paper firstly reviews the issues of global wafer logistics from wafer fabrication to IC packaging, and then proposes the solution requirements by the MDBT approach. Issues for global wafer logistics lie in two main areas. The first one is the wafer protection between transportation, handling, and storage. The current process flow for domestic and overseas wafer transportation is analyzed and the requirements of an effective and robust protection solution for transportation are proposed. The second issue is the information management between the supply chain venders. Three major functions for the information management system, including the information management for wafer transportation, receiving, and storage are suggested. With the proposed specific functional requirements, the results of this paper can be fruitful for the further design of both the wafer protection devices and the information management systems for IC foundry business.

\section{ACKNOWLEDGMENT}

This work was financially supported by the Spark Program 2013 of the Ministry of Science and Technology of PRC and Tianjin Municipal Science and Technology Commission (13ZLZLZF04400).

\section{REFERENCES}

[1] H. Sarimveis, P. Patrinos, C. D. Tarantilis, and C. T. Kiranou "Dynamic modeling and control of supply chain systems: a revewi", Computers \& Operation Research, Vol. 35, pp. 3530-3561, Nov. 2008.

[2] M. W. Ludema: "Supply Chain Configuration Management", Proceedings of IEEE/SOLI'2008, pp.2508-2513 Beijing, China, 2008.

[3] J. Chen and S. Liao: "UMC and SPIL 3D8 and 3D9 dance", Proceedings of the RosettaNet Application in Semiconductor, Hsinchu Taiwan, 2001

[4] Y. F. Lee: "RosettaNet 3D8 validation and dance", Proceedings of the RosettaNet Application in Semiconductor, Hsinchu Taiwan, 2001 .

[5] C. S. Wang: "RosettaNet implementation in semiconductor manufacturing supply chain", Proceedings of the RosettaNet Application in Semiconductor, Hsinchu Taiwan, 2001.

[6] A. W. Brown, S. Iyengar, and S. Johnsto:, "A Rational approach to model-driven development," IBM System Journal, Vol. 45, No. 3 , pp. 463-480, July 2006.

[7] J. J. Wang and D. L. Yan: "Using a hybrid multi-criteria decision aid method for information system outsourcing", Computers \& Operation Research, Vol. 34, pp. 3691-3700, Dec. 2007.

[8] D. C. Gong and Y.W. Shei: "The IDEF0 Reference Model for the Shop Flow Control Information System", Journal of the Chinese Institute of Industrial Engineers, Vol. 14, No. 1, pp. 29-37, Jan. 1997.

[9] C. Hill, R. Yates, C. Jones, and S. L. Koga: "Beyond predictable workflows: enhancing productivity in artful business processes", IBM System Journal, Vol. 45, No. 4, pp. 663-682, Oct. 2006.

[10] S. Kumaran: "Model Driven Enterprise", Proceedings of the Global Enterprise Application Integration Summit, pp. 166-180, Banf, Canada, 2004

[11] S. Kumaran, P. Bishop, T. Chao, P. Dhoolia, P. Jain, R. Jaluka, H. Ludwig, A. Moyer, and A. Nigam: "Using a Model-Driven Transformational Approach and Service-Oriented Architecture for Service Delivery Management”, IBM System Journal, Vol. 46, No. 3, pp. 513-529, July 2007

[12] Y. C. Liu: "System Analysis of Wafer Logistics Information Management for IC Packaging Foundry in Taiwan", Proceedings of IEEE/SOLI'2008, pp.2508-2513, Beijing, China, 2008 
